\title{
FUTURO DE LA DEMOCRACIA Y DEMOCRACIA DEL FUTURO
}

\author{
Andrea Greppi \\ Universidad Carlos III de Madrid
}

RESUMEN. En «Optimismo y pesimismo en la democracia» Ernesto Garzón Valdés toma como punto de partida una caracterización estándar de la democracia liberal y a partir de ella analiza las condiciones de las que depende la estabilidad de esta clase de régimen político. Por diversas razones de carácter político y social, parece ser que en nuestros días se requieren ciertas transformaciones en el modelo político vigente y por ello sugiere un decálogo de medidas que podrían contribuir a reforzar nuestras frágiles democracias. En este artículo se discute la profundidad de tales cambios y, en particular, la posibilidad y necesidad de introducir un giro deliberativo que se sitúe no sólo en el plano de los diseños institucionales, sino sobre todo en el plano de las teorías subyacentes.

Palabras clave: Ernesto Garzón Valdés, democracia liberal, democracia deliberativa.

ABSTRACT. In «Optimismo y pesimismo en la democracia» Ernesto Garzón Valdés gives a standard definition of liberal democracy and then considers the conditions that make sustainable this kind of political system. For many social and political reasons, in our days some transformations are needed, and so Garzón suggest a decalogue of measures that could reinforce our fragile democracies. This article argues about the depth of these changes, and, in particular, about the possibility and necessity of a deliberative turn that will take place not only at the level of institutional design, but especially at the level of the theory of democracy on which those institutional changes rest.

Keywords: Ernesto Garzón Valdés, liberal democracy, deliberative democracy. 


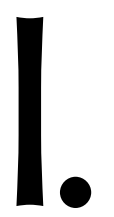

En Optimismo y pesimismo en la democracia Ernesto GARZÓN define esta forma de gobierno como un procedimiento para la toma de decisiones que asegura la paz social y garantiza el máximo de libertad en condiciones de igualdad (2003: 24). En relación con este concepto de democracia — se lee en ese texto- cabe mantener una actitud optimista si consideramos probable que se cumplan sus implicaciones prácticas, o pesimista, si pensamos lo contrario.

El resultado de este juicio de probabilidad depende de ciertas valoraciones y de una serie de circunstancias de hecho. En concreto, podremos ser optimistas con respecto al futuro de la democracia si logramos argumentar que el ideal democrático es coherente desde el punto de vista conceptual y plausible desde el punto de vista empírico. En relación con el primer tipo de condiciones, las de carácter conceptual, GARZÓN VALDÉS discute dos enfoques teóricos que mantienen una apuesta incondicional por la democracia: de un lado, el que asume el valor epistémico de los procedimientos democráticos (la referencia es a CONDORCET, entre los clásicos, y a NiNO, entre los contemporáneos); de otro, el que postula la natural sociabilidad o razonabilidad de los miembros de una sociedad democrática (los ejemplos pertinentes, en este caso, son el de HumE y el de RAWLS). A juicio de Ernesto GARZÓn, estas dos concepciones no alcanzan los objetivos previstos porque ninguna de ellas es capaz de garantizar, aunque por razones distintas, la legitimidad de los procedimientos de decisión democrática. Por eso, desde una posición «moderadamente pesimista», considera oportuno establecer alguna clase de complemento que esas dos líneas teóricas ignoran o, cuando menos, dejan en segundo plano. Su principal argumento es - como sabemos - que la democracia acaba convirtiéndose en una institución suicida cuando el ámbito de lo que puede ser decidido carece de límites. La democracia requiere, en otras palabras, un conjunto de muletas morales. Por eso la democracia contemporánea es una democracia constitucionalmente restringida, en la cual la legitimidad de las decisiones deriva del principio — que no la regla- de las mayorías.

El segundo grupo de condiciones de las que depende nuestra actitud básica de optimismo o de pesimismo en la democracia comprende aquellas condiciones que son de carácter empírico. Como ya hemos apuntado, de lo que se trata aquí es de valorar hasta qué punto es plausible, o quizá razonable, mantener la apuesta teniendo en cuenta las hipotecas que gravan sobre el funcionamiento de las democracias contemporáneas. Entre ellas, GARZÓN VALDÉS menciona la insostenibilidad de los niveles de desarrollo, el impenitente egoísmo y la incurable miopía de la especie humana, el gobierno de los expertos, la multiplicación de los instrumentos de manipulación de la conciencia, la quiebra de las garantías constitucionales y la primacía de la economía sobre la política. En relación con este segundo grupo de condiciones, y una vez descartada la actitud fatalista, que limita cínicamente las posibilidades de transformación de la realidad, propone una serie de recomendaciones que mejorarían las posibilidades de supervivencia de la democracia. El debate, como es obvio, está en saber cuál es la extensión óptima de los «ajustes» necesarios.

Sobre esta base, el decálogo de recomendaciones propuestas por GARZÓN VALDÉS gira en torno a tres núcleos fundamentales. Un primer grupo es de carácter ético, pues la supervivencia de la democracia requiere mantener alerta la conciencia y rechazar falsas ilusiones. Un segundo grupo es de carácter ético-político o, si se prefiere, de mora- 
lidad cívica, pues la democracia decae si no se mantienen vivos los valores de la tolerancia, si no ponemos remedio para evitar el cansancio de la política, y no conseguimos mantener la primacía de la esfera política sobre el ámbito de la economía. Y un tercer grupo es de carácter político-institucional o de política constitucional, pues el éxito de la democracia depende de que consigamos garantizar de manera efectiva las reglas del coto vedado, de que adoptemos las medidas necesarias de paternalismo justificable, de que consigamos eliminar las distintas formas de exclusión social, asegurar el control público en la actuación de los expertos y la transparencia de las decisiones.

II. Como bien puede verse, la propuesta de Ernesto GARZÓN no es tan «cautelosa» como dice ser y, en cambio, ofrece un listado muy exigente de indicaciones programáticas para la reforma de las instituciones. No es una propuesta de mínimos, sino de máximos, especialmente desde el punto de vista de la cultura cívica democrática. Lo que yo pregunto es si en nuestros días es suficiente seguir una estrategia como ésta, una estrategia que podemos calificar como restauradora, o si, por el contrario, no será necesario dar un paso más, un paso que nos lleve a modificar, en todo o en parte, la concepción de la democracia de la que partimos. En otros términos, lo que me pregunto es si la democracia del futuro será similar a la que conocemos, o si, por el contrario, no se verá obligada a convertirse en una cosa — al menos parcialmente-diferente. A partir de aquí propongo: 1) un diagnóstico de la situación presente, sustancialmente coincidente, pero algo más pesimista que el de GARZÓN VALDÉs; y 2) un posible remedio para los obstáculos no previstos a los que nos enfrentamos (cfr. BOBBIO, 1984). Frente a lo que GARZÓN sugiere, creo que la democracia sólo será viable en el futuro, sólo tendrá alguna clase de futuro, si logramos reinterpretarla en clave deliberativa.

III. Por lo que respecta al diagnóstico y a la posibilidad del optimismo, no me parece exagerado afirmar que asistimos a una significativa y creciente disminución de los espacios de decisión democrática. Creo además que una teoría realista, moderadamente optimista o pesimista de la democracia está obligada a asumir las consecuencias que se derivan de este proceso. Cada vez son menos los que deciden, o cuando decidimos todos lo hacemos sobre cosas menos importantes. De hecho, no es de extrañar que la democracia le importe cada vez menos a los ciudadanos. Las raíces de esta tendencia vienen de lejos y no cabe esperar remedios mágicos. Es el resultado de procesos tan extensos y profundos como son, entre otros, el vaciamiento de los instrumentos de representación política, el colapso de los mecanismos de separación y equilibrio de poderes, la descomposición del Estado legislativo, con el consiguiente déficit de legitimidad del constitucionalismo contemporáneo. Volveré enseguida sobre estas tres cuestiones.

En suma, creo que no es exagerado afirmar que nos encontramos en una situación que amenaza la quiebra de la arquitectura institucional básica de las democracias contemporáneas. Y sospecho además que para hacerle frente no basta con adoptar estrategias meramente paliativas. Pero como, conforme al ejemplo de Ernesto GARZÓN, no quiero dejarme arrastrar por el vértigo del pesimismo pasaré inmediatamente a la segunda de las observaciones que antes he anunciado, que se refiere a las distintas concepciones de la democracia y a su viabilidad en nuestro contexto político y cultural.

IV. No hace falta ser un lector demasiado perspicaz para percatarse de la desconfianza de Ernesto GARZÓN frente a las teorías deliberativas de la democracia. Las anteriores referencias a las concepciones epistémicas de la democracia y a la noción rawl- 
siana de razonabilidad son ya una prueba suficiente de ese recelo. Pero, sobre todo, basta leer sus críticas a la -que él llama-falacia de la identificación entre consenso y legitimidad y sacar las conclusiones pertinentes (GARZÓN, 1994). No es éste el lugar para reconstruir sus argumentos, ni tampoco para reflejar la diversidad de posiciones que suelen incluirse bajo el lema de la democracia deliberativa, pero sí me gustaría llamar la atención sobre un par de circunstancias que, a mi entender, deberían ser objeto de una discusión más detallada. En primer lugar, creo que ninguna propuesta (sensata) de deliberación democrática contempla un ideal en el que se deba poner en discusión todo y en todo momento, desconociendo la necesidad de fijar reglas funcionalmente análogas a las del coto vedado. Lo que aporta la perspectiva deliberativa es la idea de que tales reglas pueden evolucionar conforme a las pautas - a los procedimientos- que ellas mismas establecen. Y creo, en segundo lugar, que la tesis según la cual la estabilidad democrática requiere calificar una serie de principios básicos del orden político como materias no negociables, no implica que sobre ellas no quepa deliberación alguna.

Me detendré un instante sobre esta última cuestión. La razón más fuerte para reivindicar el giro deliberativo en el pensamiento democrático se encuentra boy en la progresiva disolución de algunas evidencias fundamentales sobre las que habían sido edificadas las teorías de la democracia dominantes en la segunda mitad del siglo XX, tanto en el campo de las teorías elitistas, como en el de las teorías participativas. En concreto, y retomando lo que se apuntó más arriba, me parece que hay al menos tres grandes ámbitos en los cuales nos enfrentamos a dificultades de la máxima intensidad y que son, ante todo, dificultades de carácter epistémico: $a$ ) en el conocimiento de los intereses y preferencias que determinan el contenido de la relación de representación política; $b$ ) en el conocimiento de las técnicas adecuadas para asegurar la separación y el equilibrio de poderes; c) en el conocimiento de la voluntad del soberano democrático, típicamente a través de las palabras de la ley. Tres ficciones - acerca de la formación de una voluntad colectiva, acerca del equilibrio virtuoso de poderes como garantía de libertad, acerca de la posible vigilancia crítica del poder por parte de la opinión pública- y tres funciones - representar, limitar, controlar - que se han vuelto especialmente inestables en el marco de sociedades complejas y cuya vigencia se halla estructuralmente vinculada al buen desarrollo de ciertos procesos de comunicación política.

Por lo demás, nadie duda de que la síntesis entre democracia y Estado de Derecho sea el mejor remedio conocido para evitar ciertos males intolerables. Lo único que se dice es que, dadas una serie de circunstancias, se ha vuelto cada vez más difícil saber cuáles son y dónde aparecen esos males. A duras penas logramos mantener vivas en la memoria algunas grandes calamidades que han marcado el desarrollo de la conciencia ética moderna (los horrores del siglo Xx, la esclavitud en sus múltiples formas, las guerras de religión, el terrorismo de Estado...), pero carecemos de instrumentos adecuados para identificar una infinidad de pequeños males cotidianos de los que depende no sólo el futuro de la democracia, sino incluso la supervivencia de la especie. Males perfectamente banales si comparados con que causan las políticas energéticas, las subvenciones a la agricultura en el primer mundo, las patentes farmacéuticas, etc., pero males relevantes.

V. No sé cuál es la forma más inteligente para gestionar el incremento de la ignorancia sobre los males menores, pero lo que sí me parece claro es que la incertidumbre afecta hoy a los presupuestos epistémicos fundamentales de nuestras teorías de la de- 
mocracia. El interés de las propuestas deliberativas está, por tanto, en la ayuda que nos ofrecen para re-construir algunas de esas evidencias, al tiempo que nos permiten de-construir otras. Dicho de otro modo, la deliberación democrática nos acompaña en el proceso de aprendizaje a través del cual llegamos a reconocer los males que nos rodean ( $c f r$. THIEBAUT, 2001). Nos proporciona pautas de racionalidad intersubjetiva para dibujar la frontera entre intereses primarios y secundarios, entre lo tolerable y lo intolerable. Y nos ayuda también a clarificar los límites de la decencia y la indecencia, así como de la atribución de responsabilidades tanto en el ámbito moral, como en el ámbito político. Por eso me atrevo a afirmar que la democracia y el consenso — en alguna de sus múltiples formas- sí son relevantes y que lo son precisamente en ese terreno fronterizo en el que perdemos pie en el suelo firme de las evidencias. Deliberamos, como explica el propio GARZÓn VALDÉS, sobre lo que no conocemos con certeza.

$\mathrm{Si}$ esto es cierto, es probable que en el futuro nos veamos obligados a introducir algunos cambios en el diseño de las instituciones democráticas, pero también —y sobre todo- en el marco de sus teorías subyacentes. Cambios que traerán algo más que simples «ajustes». En un mundo en que las evidencias tienden a desvanecerse estamos obligados a reconstruirlas sobre la marcha, pragmáticamente, con la única ayuda de lo que sabemos acerca de cómo funcionan - y de cómo podrían llegar a funcionar- los procesos de entendimiento democrático.

\section{BIBLIOGRAFÍA CITADA}

BobBio, N., 1984: Il futuro della democrazia, Torino: Einaudi.

GARZÓN VALDÉs, E., 2003: «Optimismo y pesimismo en la democracia», Claves de Razón Práctica, 131: 24-32.

- 1994: «Instituciones suicidas», Isegoría, 9: 64-128.

Thiebaut, C. 2001: «La democracia y el rechazo del daño. Sobre las condiciones públicas de la moral», en R. MAIz (ed.), Construcción de Europa, democracia y globalización, vol. 1, Universidad de Santiago de Compostela, pp. 53-66. 